\title{
Acute Hepatitis in a Child Heterozygous for the I259V MEFV Gene Variant
}

\author{
Flora Tzifi ${ }^{1}$, Philip Hawkins ${ }^{2}$, Erato Atsali ${ }^{1}$, Doxa Kotzia ${ }^{1}$, \\ Achilleas Attilakos ${ }^{1}$ \\ ${ }^{1}$ Third Department of Pediatrics, University of Athens, "Attikon" Hospital, \\ Athens, Greece; \\ ${ }^{2}$ National Amyloidosis Center, Division of Medicine, UCL Medical School, \\ Royal Free Hospital, London, United Kingdom
}

Received June 11, 2014; Accepted November 18, 2014.

Key words: Children - Familial Mediterranean Fever - Hepatitis - 1259V variant

Abstract: Familial Mediterranean Fever (FMF) is a systemic auto-inflammatory disease characterized by recurrent episodes of fever accompanied by synovial, serosal and/or cutaneous inflammation. Liver involvement has been described mainly in patients with paired FMF gene mutations, i.e. involving both alleles, and rarely in patients heterozygous for FMF mutations. These patients may present with acute or chronic hepatitis, with or without liver failure. Non-alcoholic hepatitis, mild hyperbilirubinemia, and elevation of liver enzymes of unknown etiology should also raise suspicion of FMF. Patients with FMF and liver involvement usually respond to colchicine medication. The mutation $1259 \mathrm{~V}(\mathrm{c} .775 \mathrm{~A}<\mathrm{G})$ on exon 2 of the MEFV gene has not been reported in FMF patients with liver involvement. Furthermore, among several MEFV gene variants, it has been reported so far in only one heterozygous FMF patient of Turkish ancestry presenting with abdominal pain without any hepatic complication. Herein, the second case of a FMF patient heterozygous for the above mentioned mutation is discussed. It is a male child with FMF clinical phenotype which presented two consecutively episodes of acute hepatitis during fever attacks, that spontaneously resolved. Therapeutic trial with colchicine was successful, since no other fever attacks and acute hepatitis episodes were noticed.

Mailing Address: Flora Tzifi, MD., MSc., PhD., Third Department of Pediatrics, University of Athens, "Attikon" Hospital, Rimini Str. 1, Haidari, P. C. 12462, Athens, Greece; Phone: +302 105832 228; Fax: +302 105832 229; e-mails: fltzifi@med.uoa.gr, fl_tzifi@hotmail.com 


\section{Introduction}

Familial Mediterranean Fever (FMF) is a prototypic auto-inflammatory disease characterized by recurrent febrile attacks accompanied with inflammation of the serosal membranes and of the skin and is prevalent among Mediterranean populations. The disease is due to mutations of the Mediterranean Fever Gene (MEFV), which is located in the short arm of the chromosome 16 (16p13.3) (De Sanctis et al., 2010; Zadeh et al., 2011). MEFV consists of ten exons and encodes the protein pyrin, which is involved in caspase-1 activation and consequently in interleukin-1 $\beta$ release. Pyrin is predominantly expressed in neutrophils, eosinophils, monocytes, fibroblasts and dendritic cells. Pathogenesis of auto-inflammatory diseases is associated with dysregulation of the innate immune system without any involvement of autoantigens or autoantibodies (De Sanctis et al., 2010; Zadeh et al., 2011). Several MEFV gene mutations have been described so far, mainly in the exon 10 and some others in the exons 2 and 3 . The $1259 \mathrm{~V}$ variant on exon 2 is extremely rare and published data are scarce. So far, it has been described in only one heterozygous FMF patient of Turkish ancestry presenting with abdominal pain (Ceylan et al., 2012).

Liver involvement is a rare complication of autoinflammatory syndromes and it has been described so far only in Familial Mediterranean Fever (FMF) patients (Unal et al., 2012), in one patient with hyper lgD syndrome (HIDS) (Tahara et al., 2011) and neonatal-onset chronic hepatitis, and in one child with PFAPA syndrome (periodic fever, aphtous stomatitis, pharyngitis and adenitis) and autoimmune hepatitis type II (Della Corte et al., 2010). We report the first case of a male child heterozygous for the variant $1259 \mathrm{~V}$ of the MEFV gene that presented with two consecutively acute hepatitis episodes during fever attacks which resolved spontaneously. The patient is over four years under colchicine treatment and he is free of any FMF symptoms and hepatitis episodes.

\section{Case report}

A male child aged 2.5 years old was referred to our clinic for laboratory investigation of periodical fever attacks, which appeared at the age of 6 month old. The boy is the first child of non-consanguineous parents. Delivery and perinatal period were uneventful. Genealogical tree revealed that mother's child origin was from Southern Greece and father's child origin was from Asia Minor (patient's grandfather) and Central Greece (patient's grandmother).

Episodes of recurrent fever appeared at the age of 6 month old and lasted 3-4 days, while asymptomatic periods were usually 40-50 days. Triggers for the fever attacks were infections and vaccinations. Abdominal pain was reported in three cases of previous fever episodes. The child was then referred to our clinic, at age of 2.5 years old in order to investigate periodic fever attacks. He was admitted during a fever episode at onset of fever attack and clinical examination showed a child with good general condition and normal growth. No pathological findings of 
the systems examined were noticed and specifically no pharyngitis, cervical adenitis or aphtous stomatitis were detected. Leukocyte counts, C-reactive protein and serum amyloid A protein were elevated (Table 1), while the rest routine laboratory investigation, including liver enzymes, was normal. The second day of hospitalization and illness, due to high spikes of fever, repetition of biochemical analysis revealed acute elevation of liver enzymes (Table 1). There was no abdominal pain or palpable liver at clinical examination. Liver ultrasound showed no echogenicity abnormalities. An extensive laboratory investigation was conducted for hepatitis differential diagnosis, which excluded several causes of hepatitis (Table 2) and since no prior medication was reported, we hypothesized that acute liver enzymes elevation could be attributed to the underlying autoinflammatory disease.

Serum liver enzymes returned to normal values without treatment within fifteen days. One month later, the child was admitted again for fever attack and a milder liver enzyme elevation was observed, which resolved spontaneously. It should be noted that during asymptomatic periods and in previous episodes of fever no liver dysfunction was found.

Table 1 - Biochemical investigation of the patient during two fever attacks

\begin{tabular}{lccccc}
\hline $\mathbf{1}^{\text {st }}$ admission & $1^{\text {st }}$ day & $2^{\text {nd }}$ day & $3^{\text {rd }}$ day & $5^{\text {th }}$ day & Normal values \\
\hline Leukocyte count & 14610 & 12820 & 11690 & 9530 & $4000-11000 \mathrm{k} / \mu \lambda$ \\
C-reactive protein & 17 & 104 & 119 & 24 & $0.0-6.0 \mathrm{mg} / \mathrm{l}$ \\
AST/SGOT & 30 & $\mathbf{2 0 3 9}$ & $\mathbf{3 1 9}$ & 41 & $15-48 \mathrm{U} / \mathrm{l}$ \\
ALT/SGPT & 22 & $\mathbf{1 0 8 1}$ & $\mathbf{6 8 8}$ & $\mathbf{2 3 7}$ & $13-40 \mathrm{U} / \mathrm{l}$ \\
$\gamma$-GT & 17 & $\mathbf{2 4 1}$ & $\mathbf{2 9 7}$ & $\mathbf{1 9 4}$ & $9-50 \mathrm{U} / \mathrm{l}$ \\
Tbil/Dbil & - & $\mathbf{2 . 1 7 / 1 . 3 7}$ & $0.9 / 0.4$ & $0.36 / 0.14$ & $\begin{array}{c}\text { Tbil: } 0.2-1.0 \mathrm{mg} / \mathrm{dl} \\
\end{array}$ \\
Serum amyloid & $\mathbf{1 4 9}$ & & & & Dbil: $<0.3 \mathrm{mg} / \mathrm{dl}$ \\
protein & & & & & $0.0-6.4 \mathrm{mg} / \mathrm{l}$ \\
\hline $2^{\text {nd }}$ admission & $1^{\text {st }}$ day & $2^{\text {nd }}$ day & $3^{\text {rd }}$ day & $10^{\text {th }}$ day & Normal values \\
\hline Leukocyte count & 12340 & 11250 & 14210 & 6370 & $4000-11000 \mathrm{k} / \mu \lambda$ \\
C-reactive protein & 19.6 & 54.5 & 217 & 60 & $0.0-6.0 \mathrm{mg} / \mathrm{l}$ \\
AST/SGOT & 35 & 38 & $\mathbf{1 8 7}$ & 38 & $15-48 \mathrm{U} / \mathrm{l}$ \\
ALT/SGPT & 19 & 23 & $\mathbf{1 4 9}$ & $\mathbf{1 0 7}$ & $13-40 \mathrm{U} / \mathrm{l}$ \\
$\gamma$-GT & 22 & 22 & 48 & $\mathbf{6 8}$ & $9-50 \mathrm{U} / \mathrm{l}$ \\
Tbil/Dbil & $0.37 / 0.11$ & $0.54 / 0.15$ & $0.5 / 0.2$ & $0.1 / 0.1$ & Tbil: $0.2-1.0 \mathrm{mg} / \mathrm{dl}$ \\
& & & & & Dbil: $<0.3 \mathrm{mg} / \mathrm{dl}$ \\
Serum amyloid & $\mathbf{2 0 0}$ & & & & $0.0-6.4 \mathrm{mg} / \mathrm{l}$ \\
Protein & & & & & \\
\hline
\end{tabular}

This table shows liver enzymes elevation during two fever attacks while the patient was hospitalized in our clinic. The interval between the episodes was one month. Acute elevation of the liver enzymes was noticed but they returned to normal in few days. Serum amyloid protein was elevated too.AST/SGOT - aspartic transaminase;ALT/SGPT - alanine transaminase; $\gamma$-GT - $\gamma$-glutamyl-transpeptidase; Tbil - total bilirubin; Dbil - direct bilirubin 
Table 2 - Differential diagnosis of hepatitis in our patient

Infectious agents: hepatitis A, B, C, E, CMV, EBV, Toxoplasma gondii, HSV 1, 2

Autoimmune hepatitis: ANA, anti-dsDNA, anti-LKM, SMA autoantibodies Immunologic evaluation - Immunoglobulins (IgA, $\lg M$, $\lg G, \lg \mathrm{D})$

- Lymphocyte subpopulations

- Antibody response to tetanus, haemophilus, pneumococcus

- Complement (C3, C4)

Metabolic diseases (blood and urine amino acids, urine organic acids)

A1-antithrypsin deficiency

Wilson disease

\section{Gilbert syndrome}

This table shows the laboratory investigation performed in order to seek for other causes of hepatitis. All findings were negative. CMV - cytomegalovirus; EBV - Epstein-Barr virus; HSV 1, 2 - herpes 1, 2 viruses;ANA - antinuclear antibodies; anti-dsDNA - antibodies against double-stranded DNA; anti-LKM - anti-liver kidney microsome antibodies; SMA - smooth muscle antibodies

Patient's clinical and laboratory findings led to genetic test for periodic autoinflammatory syndromes. Genetic evaluation was held at the National Amyloidosis Centre (Royal Free Hospital, London). MEFV, TNFRSF1A and MVK genes were analyzed by polymerase chain reaction and automated sequencing. Mutations analysis revealed that the patient was heterozygous for the variant $1259 \mathrm{~V}$ $(775 \mathrm{~A}<\mathrm{G})$ of the MEFV gene. No other mutations at all exons of the MEFV gene were identified. Furthermore, screening for mutations at exons 9 and 11 of the MVK gene and at exons 2-3, 4-5, 6-7 and introns 2, 4 and 6 of the TNFRSF1A gene was negative.

Since the patient was heterozygous for MEFV gene mutations, colchicine treatment was initiated, $0.5 \mathrm{mg}$ daily, as a therapeutic trial in order to establish FMF diagnosis. The child has received colchicine for four years without side effects and thereafter recurrent fever attacks and acute hepatitis episodes have been resolved.

\section{Discussion}

Liver involvement in homozygous or heterozygous patients with FMF has been described previously, mainly as case reports (Unal et al., 2012). One female patient with Sjogren syndrome and FMF and two pediatric patients with FMF have been reported to present with acute hepatitis during fever episodes that resolved without specific treatment (Neequaye and Jelly, 1994; Migita et al., 2007). In a recent study in a pediatric population with FMF, liver involvement has been described as abnormal liver enzyme elevation of unknown etiology, cholelithiasis, hepatomegaly, chronic liver disease with fibrosis, Budd-Chiari syndrome, and acute hepatitis. Several MEFV alleles have been detected in these patients, of which the M694V allele has the highest frequency (Unal et al., 2012). The pathophysiological 
mechanism of acute septic liver injury in FMF comprises the role of inflammatory cytokines like tumor necrosis factor alpha and interleukin 6. This acute liver injury may progress to chronic hepatitis and to cirrhosis especially in undiagnosed cases. Histological examination of the liver biopsy in these patients usually has the findings of non-specific reactive hepatitis (Unal et al., 2012). All FMF children with liver injury respond to colchicine treatment (Unal et al., 2012), as well as the child presented.

Our patient fulfills the criteria required for FMF diagnosis. According to Tel Hashomer criteria (Livneh et al., 1997), the child has two major criteria (recurrent fever and abdominal pain, response to colchicine treatment) that are in favor of FMF diagnosis. According to Yalçinkaya et al. (2009) pediatric criteria, the child had fever (axillary temperature $>38^{\circ} \mathrm{C}$, more than 3 attacks, duration 3-4 days) and abdominal pain, that is 2 of the 5 criteria (the other three are thoracic pain, synovitis, family history) required for FMF diagnosis. Genetic testing results though did not reveal any classical FMF mutation and excluded HIDS (hyper IgD syndrome) and tumor necrosis factor receptor-associated periodic syndrome (TRAPS). Published data regarding the $1259 \mathrm{~V}$ allele are scarce and only one heterozygous FMF patient of Turkish ancestry presenting with abdominal pain has been reported so far (Ceylan et al., 2012). To our knowledge, this allele has not been reported in the Greek population but the origin of the grandfather's child from Asia Minor could explain the detection of this allele in the case presented (Giaglis et al., 2007). Heterozygocity, despite sequencing of all MEFV exons, could be attributed to the fact that current mutation detection techniques cannot reveal disease causing mutations in noncoding intronic areas of the gene or in a gene region that regulates messenger RNA expression or splicing (Zadeh et al., 2011). It is known that genetic testing for FMF is of weak diagnostic value in many cases as well as in our patient.

In our case, all other causes of hepatitis were excluded, although no liver biopsy was performed. Acute hepatitis resolved without specific treatment as it has been described in previous cases of FMF.Therefore, this is the first case of an individual heterozygous for the variant $1259 \mathrm{~V}$ which presents with: a) the classical clinical manifestations of FMF early in life and b) liver involvement, which is considered as a rare complication of FMF.A putative explanation of the case reported could be the fact that other unknown modifier genes or epigenetic factors are involved in the pathogenesis of FMF resulting in the heterogeneity of the clinical phenotype among these patients. Several studies have implicated different genes (Rüstemoglu et al., 2011) but large longitudinal studies are required in order to clarify this topic.

Acknowledgements:The authors thank to Dr. Olga Vougiouka, Director of the Pediatric Rheumatology Department of Children's Hospital "Aglaia Kyriakou", for her contribution in patient's care and management. We also thank Hadija Troger, Technician of National Amyloidosis Center, Royal Free Hospital, London, for carrying out molecular analysis of the patient. 


\section{References}

Ceylan, G. G., Ceylan, C., Ozturk, E. (2012) Frequency of alterations in the MEFV gene and clinical signs in familial Mediterranean fever in Central Anatolia, Turkey. Genet. Mol. Res. 7, 1185-1194.

De Sanctis, S., Nozzi, M., Del Torto, M., Scardapane, A., Gaspari, S., de Michele, G., Breda, L., Chiarelli, F. (2010) Autoinflammatory syndromes: diagnosis and management. Ital. J. Pediatr. 36, 57.

Della Corte, C., Ranucci, G., Tufano, M., Alessio, M., lorio, R. (2010) Autoimmune hepatitis type 2 arising in PFAPA syndrome: coincidences or possible correlations? Pediatrics 125, e683-e686.

Giaglis, S., Papadopoulos, V., Kambas, K., Doumas, M., Tsironidou, V., Rafail, S., Kartalis, G., Speletas, M., Ritis, K. (2007) MEFV alterations and population genetics analysis in a large cohort of Greek patients with familial Mediterranean fever. Clin. Genet. 71, 458-467.

Livneh, A., Langevitz, P., Zemer, D., Zaks, N., Kees, S., Lidar, T., Migdal, A., Padeh, S., Pras, M. (1997) Criteria for the diagnosis of familial Mediterranean fever. Arthritis Rheum. 40, 1879-1885.

Migita, K., Abiru, S., Tanaka, M., Ito, M., Miyashita, T., Maeda, Y., Koga, T., Nakamura, M., Komori, A., Yatsuhashi, H., Ida, H., Eguchi, K., Hirayama, K., Yasunami, M., Ishibashi, H. (2007) Acute hepatitis in a patient with familial Mediterranean fever. Liver Int. 28, 140-142.

Neequaye, J., Jelly, A. E. (1994) Acute hepatitis in recurrent hereditary polyserositis (familial Mediterranean fever). J. Trop. Pediatr. 40, 243-245.

Rüstemoglu, A., Gumus-Akay, G., Yigit, S., Tasliyurt, T. (2011) Analysis of common MDR1 (ABCB1) gene C1236T and C3435T polymorphisms in Turkish patients with familial Mediterranean fever. Genet. Mol. Res. 14, 3411-3420.

Tahara, M., Sakai, H., Nishikomori, R., Yasumi, T., Heike, T., Nagata, I., Inui, A., Fujisawa, T., Shigematsu, Y., Nishijima, K., Kuwakado, K., Watabe, S., Kameyama, J. (2011) Patient with neonatal-onset chronic hepatitis presenting with mevalonate kinase deficiency with a novel MVK gene mutation. Mod. Rheumatol. 21, 641-645.

Unal, F., Cakir, M., Baran, M., Arıkan, C., Yuksekkaya, H. A., Aydoğdu, S. (2012) Liver involvement in children with familial Mediterranean fever. Dig. Liver Dis. 44, 689-693.

Yalçinkaya, F., Ozen, S., Ozçakar, Z. B., Aktay, N., Cakar, N., Düzova, A., Kasapçopur, O., Elhan, A. H., Doganay, B., Ekim, M., Kara, N., Uncu, N., Bakkaloglu, A. (2009) A new set of criteria for the diagnosis of familial Mediterranean fever in childhood. Rheumatology (Oxford) 48, 395-398.

Zadeh, N., Getzug, T., Grody, W.W. (2011) Diagnosis and management of familial Mediterranean fever: integrating medical genetics in a dedicated interdisciplinary clinic. Genet. Med. 13, 263-269. 